\title{
Identifying the Challenges and Requirements of Enterprise Architecture Frameworks for IoT Systems
}

\author{
Filip Vanhoorelbeke ${ }^{1}$, Monique Snoeck $^{2}$, Estefanía Serral ${ }^{1 \varpi}$ \\ ${ }^{1}$ KU Leuven, Warmoesberg, 26, Brussels 1000, Belgium \\ ${ }^{2}$ KU Leuven, Naamsestraat 69, Leuven, 3000, Belgium
}

\begin{abstract}
Enterprise Architecture Frameworks (EAFs) have been around since the last decades of the 20th century. They are a proven practice to analyze, describe, organize, implement and manage changes in the global architecture of an enterprise's data, processes, applications and technology. Recently, new promising technologies such as big data, machine learning, and the always-and-everywhere connected Internet of Things (IoT), have made their way into all sorts of business-generating activities. The vast number of possible connectable devices, with almost infinite useful applications throughout an enterprise such as operations, human resource management, communications, and customer service, demonstrates the holistic nature of IoT. Because of that, making use of IoT cannot be treated in isolation, but should be integrated in all aspects of Enterprise Architecture. Therefore, this paper identifies the main architectural challenges and derived requirements of IoT systems for an EAF. A literature study and a questionnaire aimed at industry EA experts have been used as main data sources.
\end{abstract}

Keywords: Enterprise Architecture, Enterprise Architecture Frameworks, the Internet of Things, IoT.

\section{Introduction}

Enterprise Architecture Frameworks (EAFs) have been around since the last decades of the 20th century, a time where memory and storage were very costly for an enterprise. However, technology has drastically evolved over the years, bringing along new types of business processes and data applications, and making unclear whether current EAFs are prepared to facilitate the management of such evolving technologies, data, and processes. One of the promising new technologies that finds itself in the adoption phase is the Internet of Things (IoT) . More and more does IoT find its way in the daily lives of our society, opening a whole world of business opportunities.

IoT is still often seen as not more than a money-bleeding gimmick which has yet to prove itself as being a potential value-adding asset. It remains to be seen if the opportunities of IoT can outweigh the challenges it faces, or it becomes a liability instead of delivering business value. This is where an EAF could help by providing methodologies for constructing a to-be architecture including IoT, reference material to aid the process of building such architecture, and means to inform and collect input from different 
kinds of stakeholders on different abstraction levels. The question is: can current EAFs support the new challenges that IoT brings? This paper will therefore focus on identifying the challenges that IoT brings for EAFs and to derive what requirements should be addressed to deal with those requirements.

The rest of this paper is organized as follows. Section 2 presents the methodology used in this paper to answer the research questions along with related works about EAFs, IoT, and EAFs specifically designed with IoT in mind. In Section 3, the results of the questionnaire and the interviews are elaborated on. Section 4 presents the identified IoT challenges and consequently the requirements for an EAF. Finally, this paper finishes with a discussion and possible future research in Section 5.

\section{Research method and related work}

The methodology used in this paper is twofold. First, a literature study was performed to understand the current state of the art concerning IoT and EAFs, including those explicitly aimed at IoT. For the literature, around 30 papers on the topics of IoT, EAFs, EAFs for IoT, Enterprise modeling, IoT architecture, and Enterprise Architecture Management have been thoroughly researched using Google Scholar. Also, multiple surveys such as [1] have been used as source of input.

In the literature study, the challenges that IoT brings are identified. Based on these challenges, requirements that an EAF needs to satisfy to support IoT are determined. To identify the challenges, research was done on existing papers that focus on IoT challenges, e.g. [2-6]; papers that focus on EAFs' challenges, e.g. [7-9]; and papers that overlap in both topics, e.g. [10-13]. From these papers, a list of challenges was derived.

To allow for a more holistic approach towards the identification of requirements and challenges, a questionnaire aimed at Enterprise Architects was performed. Questions were asked to determine: 1) the professional profile of the expert, 2) strengths and weaknesses of the EAFs in use, 3) EAFs support for IoT. We also asked the experts if they would be willing to be interviewed. These interviews allowed for additional context on some of the responses that were given in the questionnaire. Both the literature study and the responses of the experts were used to refine the identified IoT challenges and requirements.

\section{Experts' Responses}

A total of 35 EAF experts answered the questionnaire of which 4 were interviewed. Their demographic information is provided in Table 1. Figure 1 shows the EAFs that were most used by these experts at their workplace. On the question whether IoT is being used or is planned on being used within the company of the experts, around $62 \%$ responded with 'definitely yes', 19\% with 'probably yes', and $12 \%$ with 'might or might not'. Only $8 \%$ of the questioned experts indicate that the company they work at is not planning on using IoT to deliver business value. Over half of the respondents $(58 \%)$ do not believe that new EAFs that would be specifically designed for IoT scenarios would be different from the existing EAFs, while $42 \%$ believes the opposite. While the opinion of the experts is leaning towards the former, it is clear that there is 
no firm consensus on this topic yet. According to the experts who think current EAFs are not entirely suited for IoT, EAFs lack, among others: deployment models, device management, and reference architectures. The experts who think current EAFs are suited often cite that IoT is a technology as any other and that the EAFs they use have a high enough abstraction level to fit in new technologies.

Table 1. Demographic info

\begin{tabular}{lrr}
\hline Demographic & Count & $\%$ \\
\hline Number of respondents & 35 & \\
Average age & 47.17 & \\
Years of experience in EA field & & \\
$10+$ & 24 & $69 \%$ \\
$5-10$ & 4 & $11 \%$ \\
$2-5$ & 4 & $11 \%$ \\
$0-2$ & 3 & $9 \%$ \\
Years of working experience & & \\
$10+$ & 33 & $94 \%$ \\
$5-10$ & 1 & $3 \%$ \\
$2-5$ & 1 & $3 \%$ \\
\hline \multicolumn{2}{l}{ (rounded to nearest integer, not all percentages add up to } \\
$\quad 100 \%)$ &
\end{tabular}

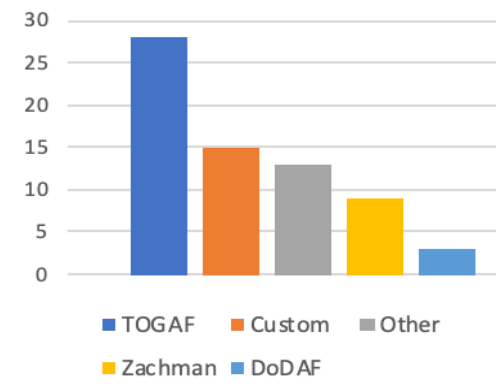

Fig. 1. EAFs in use at the respondents' company

\section{IoT Challenges and Requirements for an EAF}

This section presents the most important challenges that stem from the literature or were raised by the experts that were questioned. To define a set of requirements that an EAF needs to be able to address, the challenges that IoT brings need to be analyzed. In the following we summarize the challenges and the extracted requirements:

Multiple stakeholder perspectives: In In an IoT system multiple stakeholders geographical distant and with a different level of interest and expertise often collaborate $[3,14]$. Decision making can be a lengthy and a challenging process with multiple levels each required to approve a particular decision. Therefore, aligning the business objectives with IoT can be challenging from a governance perspective. To deal with this challenge, the EAF needs to provide a means of governance.

Primary requirements: life cycle management, governance, stakeholder management Derived requirements: change management, reference architecture

Data management: Devices of all kind, with diverse capabilities for IoT, can generate enormous amounts of data. This heterogeneous data needs to be processed and stored, often in a distributed way [15]. The management and storage of this data is a big challenge because current architectures are not prepared yet for this amount of information [2]. To address this challenge, an EAF needs to support adequate data management, data analytics, and device management.

Primary requirements: data management, complexity management 
Derived requirements: change management, integration \& interoperability, security, scalability \& maintainability

Privacy and Security: Since many stakeholders are involved, a robust security strategy is required to ensure data privacy. The data that finds itself within the IoT system needs to be stored safely and with respect for privacy of the stakeholders. Especially in a European context, where the General Data Protection Regulation (GDPR, Regulation 2016/679) is in place since May 25, 2018, the way how data is handled is essential since failing to meet the requirements could have a severe financial impact on an enterprise.

Because of the severe impact a breach could have on data integrity and privacy, security is one of the most critical challenges that an EA faces [16].

Primary requirements: security, privacy

Derived requirements: change management, complexity management, risk management

Integration: Service-oriented architectures, which is the case with IoT, need integrations on multiple levels with a potentially enormous amount of different heterogeneous systems. A challenge is that different objects need to be able to work together in a standardized way. Today there is a lack of standardization and semantic interoperability on multiple levels. Because of the complexity of the involved systems, an EAF suited for IoT would benefit from reference architectures. Being able to model IoT-specific capabilities would be a valuable capability that an EAF could offer. To be able to react quickly, proper change management along with an agile supporting lifecycle management are required. Finally, it is also important to talk about the challenge of integrating IoT in an already existing architecture.

Primary requirements: integration \& interoperability, complexity management, lifecycle management, reference architecture

Derived requirements: change management, governance, risk management, stakeholder management

Financial Cost: Implementing a new technology such as IoT brings along important investments. Estimating potential long-term effects of the implementation of an IoT system can be complicated. The uncertain nature of IT, the immaturity of IoT, and the rapid pace of technological advances are challenges that need proper risk assessment and control [2-17]. An EAF should be able to provide means to estimate the needed financial investment to develop the to-be EA.

Primary requirements: risk management

Derived requirements: change management, lifecycle management, stakeholder management

Reliability, performance, and scalability: Fallback systems are no unnecessary luxury. Not only high-availability is a must, but also the performance of communication between the different components is a crucial factor [16]. In addition, an EAF for IoT systems needs to address the fact that IoT systems can have a large scale of operations that require abstractions at different technology layers [16-18]. 
Primary requirements: quality management, scalability \& maintainability Derived requirements: change management, integration \& interoperability, lifecycle management, risk management

EA Design Process: Some EAFs have extensive reference architectures to aid the design of an architecture. It can be challenging to find adequate reference material that fits the wanted scenario. The reference models of these reference architectures can serve as templates and best-practices on how to build the system on an enterprise-scale [19]. Another challenge during the process of conceiving an architecture is that of the continuously changing business requirements.

Primary requirements: change management, lifecycle management, reference architecture

Derived requirements: complexity management

\section{Conclusions, Discussion, and Future Research}

The challenges and derived requirements of IoT that have been analyzed in this paper indicate that the needs of an IoT system are of a specific nature. Integration and interoperability are of the most important aspects that an EAF for IoT systems would need to address. Also, when addressing integration challenges, being able to model IoT-specific capabilities such as cloud integrations, wireless communication, and autonomous functionalities, would be a valuable EAF feature. For this, the requirement of architecture modeling is very important. Other important requirements that have been identified related to the integration challenge are security management, lifecycle management, change management and complexity management.

Remarkably, none of the experts stated explicitly the usability of a framework as an important requirement for an EAF. Usability should be a factor in the decision-making process on whether an existing EAF currently in use should be extended for IoT scenarios within a company or another more suited EAF should be chosen. However, the requirements of change management, lifecycle management, and risk management could be expanded to include this topic. Being able to quickly and effectively introduce and implement a new EAF, which could have a steep learning curve, into an enterprise is a factor that should not be underestimated. Depending on what EAF one is familiar with, and how deep one's knowledge is about an EAF, it is likely that one comes to a different conclusion on which EAF to finally use.

Future research would require deepening and refining the specifications of the identified requirements, and executing a complete comparison of established EAFs such as TOGAF, the Zachman Framework, DoDAF, and the UAF versus newer EAFs specifically aimed at IoT such as ESARC. These EAFs could be compared on basis of the identified requirements stated in this paper to determine the level of suitability of each EAF for IoT systems and to identify particular gaps that should be addressed to improve this suitability. Specifically, because the lack of best practices and reference architectures is a recurring theme amongst the inquired experts and current EAFs, future research in this area would be highly beneficial for EAFs in regard to IoT. 


\section{References}

[1] A. Gluhak et al., "A Survey on Facilities for Experimental Internet of Things Research," IEEE Commun. Mag., vol. 49, no. 11, pp. 58-67, 2014.

[2] I. Lee and K. Lee, "The Internet of Things (IoT): Applications, investments, and challenges for enterprises," Bus. Horiz., vol. 58, no. 4, pp. 431-440, 2015.

[3] R. van Kranenburg and A. Bassi, "IoT Challenges," Commun. Mob. Comput., vol. 1, no. 1, p. 9, 2012.

[4] A. Reyna, C. Martín, J. Chen, E. Soler, and M. Díaz, "On blockchain and its integration with IoT. Challenges and opportunities," Futur. Gener. Comput. Syst., vol. 88, no. 2018, pp. 173-190, 2018.

[5] A. Al-Fuqaha, M. Guizani, M. Mohammadi, M. Aledhari, and M. Ayyash, "Internet of Things: A Survey on Enabling Technologies, Protocols, and Applications," IEEE Commun. Surv. Tutorials, vol. 17, no. 4, pp. 2347-2376, 2015.

[6] N. Dyess, "Six IoT challenges, solutions," Control Eng., no. October, p. 21, 2018.

[7] D. Romero and F. Vernadat, "Enterprise information systems state of the art: Past, present and future trends," Comput. Ind., vol. 79, pp. 3-13, 2016.

[8] S. Buckl and C. M. Schweda, "On the State-of-the-Art in Enterprise Architecture Management Literature," Tech. Rep., p. 144, 2011.

[9] R. Giachetti, Design of Enterprise Systems. 2010. CRC Press.

[10] Z. Pourzolfaghar, V. Bastidas, and M. Helfert, "Standardisation of enterprise architecture development for smart cities," J. Knowl. Econ., 2019.

[11] K. Dar, A. Taherkordi, H. Baraki, F. Eliassen, and K. Geihs, "A resource oriented integration architecture for the Internet of Things: A business process perspective," Pervasive Mob. Comput., vol. 20, pp. 145-159, 2015.

[12] R. Baxter, N. Hastings, A. Law, and E. J. Glass, Architecting the Internet of Things, vol. 39, no. 5. 2011.

[13] A. Zimmermann, R. Schmidt, K. Sandkuhl, M. Wißotzki, D. Jugel, and M. Möhring, "Digital enterprise architecture-transformation for the internet of things," EDOCW 2015, no. October, pp. 130-138, 2015.

[14] P. Patel and D. Cassou, "Enabling high-level application development for the Internet of Things," J. Syst. Softw., vol. 103, pp. 62-84, 2015.

[15] M. A. Razzaque, M. Milojevic-Jevric, A. Palade, and S. Cla, "Middleware for internet of things: A survey," IEEE Internet Things J., vol. 3, no. 1, pp. 70-95, 2016.

[16] I. S. Udoh and G. Kotonya, "Developing IoT applications: challenges and frameworks," IET Cyber-Physical Syst. Theory Appl., vol. 3, no. 2, pp. 65-72, 2017.

[17] R. G. Fichman, M. Keil, and A. Tiwana, "Beyond Valuation: Options Thinking in IT Project Management," Calif. Manage. Rev., vol. 47, no. 2, 2005.

[18] M. R. Abdmeziem, D. Tandjaoui, and I. Romdhani, "Architecting the Internet of Things: State of the Art," no. July, p. 351, 2015.

[19] D. Guinard, V. Trifa, F. Mattern, and E. Wilde, Architecting the Internet of Things, no. January. 2011. 